\title{
A Gas Sensor with BLE connectivity for Wearable Applications ${ }^{+}$
}

\author{
Alessandro Zompanti ${ }^{1}$, Marco Santonico ${ }^{1}$, Luca Vollero ${ }^{2}$, Simone Grasso ${ }^{1}$, Anna Sabatini 1, \\ Federico Mereu ${ }^{1}$, Arnaldo D'Amico ${ }^{3}$ and Giorgio Pennazza ${ }^{1, *}$ \\ 1 Unit of Electronics for Sensor Systems, Department of Engineering, \\ Campus Bio-Medico University of Rome, Via Alvaro del Portillo, 21-00128 Rome, Italy; \\ a.zompanti@unicampus.it (A.Z.); m.santonico@unicampus.it (M.S.); s.grasso@unicampus.it (S.G.); \\ a.sabatini@unicampus.it (A.S.); f.mereu@unicampus.it (F.M.) \\ 2 Unit of Computational Systems and Bioinformatics, Department of Engineering, Campus Bio-Medico \\ University of Rome, Via Alvaro del Portillo, 21-00128 Rome, Italy; 1.vollero@unicampus.it \\ 3 Department of Electronic Engineering, Tor Vergata University of Rome, Via del Politecnico, \\ 1-00133 Rome, Italy; damico@eln.uniroma2.it \\ * Correspondence: g.pennazza@unicampus.it; Tel.: +39-06-225418465 \\ † Presented at the Eurosensors 2018 Conference, Graz, Austria, 9-12 September 2018.
}

Published: 21 November 2018

\begin{abstract}
The technological development of the last few years in the field of integrated electronic components has encouraged the use of wearable electronic devices. In the biomedical field, this improvement allows the registration and analysis of numerous values, starting from environmental parameters up to the vital parameters of a subject, without interfering with the normal daily activities of the individual. In this context, the present work is focused on the design, development and evaluation of a low power wearable and wireless electronic interface able to acquire and transmit signals generated by a gas sensor, based on electrochemical technology, to monitor air quality through the measurement of $\mathrm{O} 2$ and $\mathrm{CO} 2$ concentration. Among the existing wireless technologies, it was decided to use Bluetooth Low Energy (BLE) as it allows data transmission to multiple types of external devices, such as PCs and smartphones with low power consumption.
\end{abstract}

Keywords: wearable sensors; low power electronics; biomedical applications; air quality; Bluetooth Low Energy (BLE)

\section{Introduction}

In a real life scenario, the monitoring of selected physico-chemical parameters could support the supervising of an individual activity allowing the simultaneous registration of data from the environment and on the subject health status [1,2]. The continuous monitoring of environmental parameters in a single device which is also wearable, wireless and low-power, could represent a brick to be added to the future realization of a wearable system formed by multiple devices. This system could allow the simultaneous detection and integration of environmental and vital parameters, to be used for an effective application of preventive medicine [3]. Very often, indeed, the health status of a subject is given by a series of vital parameters instead of a single one. Besides, the monitoring of environmental data such as gas concentration, temperature and relative humidity, could help identifying potential warning conditions giving rise to uncomfortable or warming situations. Oxygen and Carbon dioxide concentrations in air are crucial for indoor air quality, and practical indications can be found in literature about thresholds [4] to be frequently monitored. This study presents a sensor system, 
which is able to perform electrochemical analysis of $\mathrm{O}_{2}$ and $\mathrm{CO}_{2}$ concentrations in the environment [5], with very low power consumption and in wireless modality.

\section{Experimental}

The novel electrochemical sensing system is based on a previous developed device [5] and includes a Screen-Printed Electrode probe (SPE, DRP-250BT, Metrohm, Herisau, Switzerland), which acts as an electrochemical cell, a new dedicated electronic interface driving the electrode, a microcontroller for input/output signals and a BLE module to transmit data to an external device. The electronic interface was properly designed to achieve low power consumption (about $80 \mathrm{~mW}: 3.6 \mathrm{~V}, 22 \mathrm{~mA}$ ) and reduced size (about $12 \mathrm{~cm}^{2}$ ) (Figure 1).

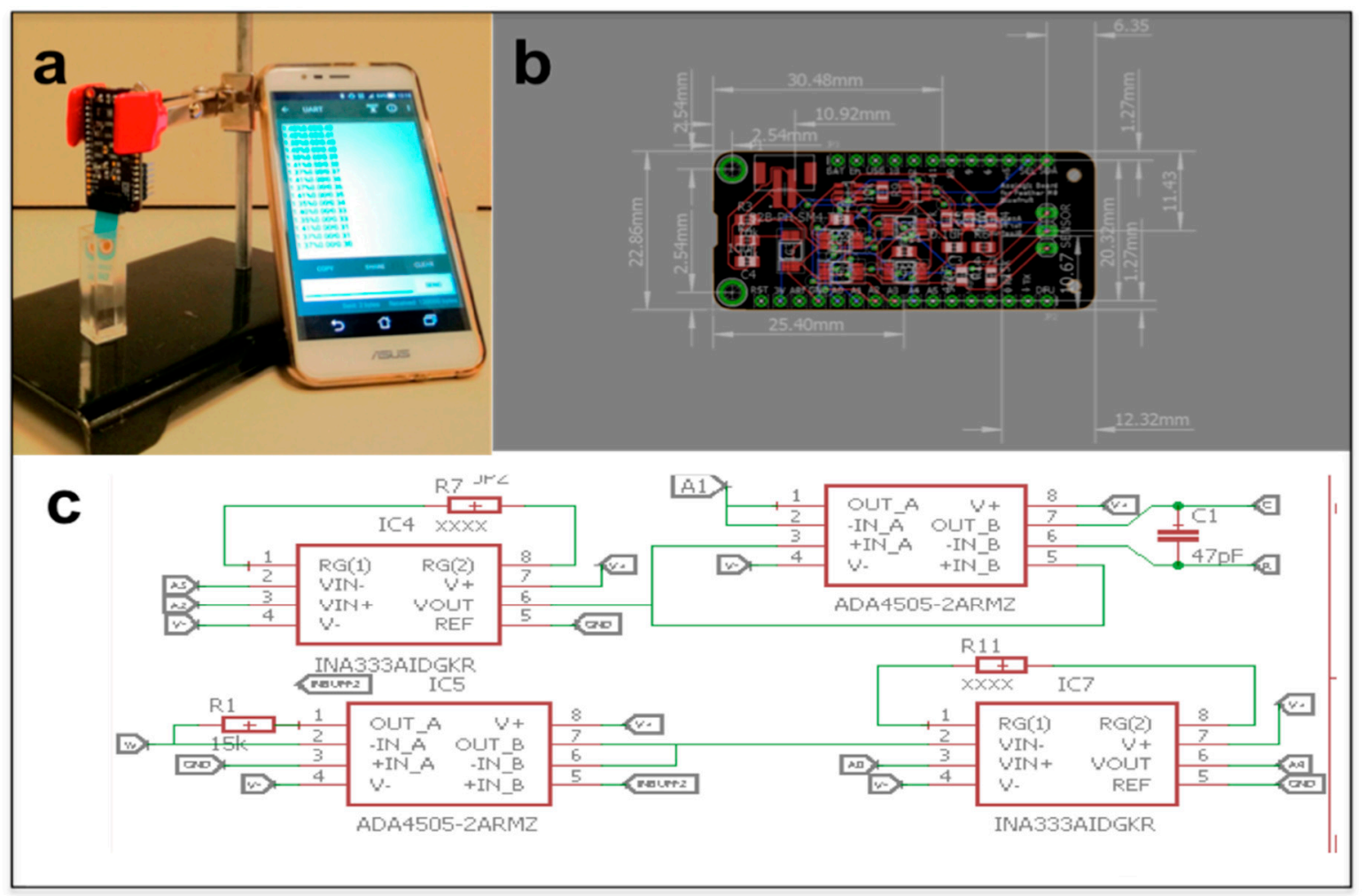

Figure 1. Developed sensor system analysing a liquid sample and transmitting data to a smartphone using a BLE connection: (a) experimental set-up using a smartphone; (b) layout of the electronic interface; (c) schematic of the electronic interface between the sensor and the controller.

Different mixtures of $\mathrm{N}_{2}$ and $\mathrm{O}_{2}$ or $\mathrm{CO}_{2}$ were fluxed into a $4 \mathrm{~mL}$ solution of saline and distilled water (in a ratio of 1:20) for $30 \mathrm{~s}$ to dissolve the gas into the liquid medium; the liquid sample was subsequently analyzed using the electrochemical sensing system. Tested $\mathrm{O}_{2}$ and $\mathrm{CO}_{2}$ concentrations ranged from $0 \%$ to $100 \%$ by steps of $10 \%$. A sinusoidal input signal $(0.2 \mathrm{~Hz}$, $\pm 2 \mathrm{Vpp}$ ) was applied to the electrochemical cell and the current generated by induced oxi-reduction phenomena was converted into a voltage and acquired as the output signal with a sampling rate of $100 \mathrm{~Hz}$; thus the output signals consists of a cyclic voltammogram of 500 points. Data were sent to a smartphone using BLE connection. The entire output signal is treated as a fingerprint of the sample using a multivariate data analysis technique: Partial Least Square Discriminant Analysis (PLS-DA), cross-validated via the Leave-One-Out criterion. 


\section{Results}

The sensing system has showed the ability to measure $\mathrm{O}_{2}$ and $\mathrm{CO}_{2}$ concentrations: PLS-DA models calculated on the calibration data set of each compound have shown Root Mean Square Error in Cross Validation (RMSECV), of $3.39 \%$ and $0.47 \%$ for $\mathrm{CO}_{2}$ and $\mathrm{O}_{2}$ respectively. The calibration results obtained with this new low-power and wireless system has been compared with the performance given by the first prototype [4]. The order of magnitude of the RMSECV is the same.

Moreover, looking at $\mathrm{CO}_{2}$ there is an increasing of the estimated detection limit of about $1.4 \%$, while $\mathrm{O}_{2}$ detection limit has been lowered of 4 times. The interesting RMSECVs obtained via multivariate data analysis techniques suggest that the different concentration levels should be visible also by comparing the output voltammograms given by each concentration step tested during the calibration phase. In Figure 2 the output $\mathrm{O}_{2}$ signals for steps of $10 \%$ are shown. The registered curves are evidently different.
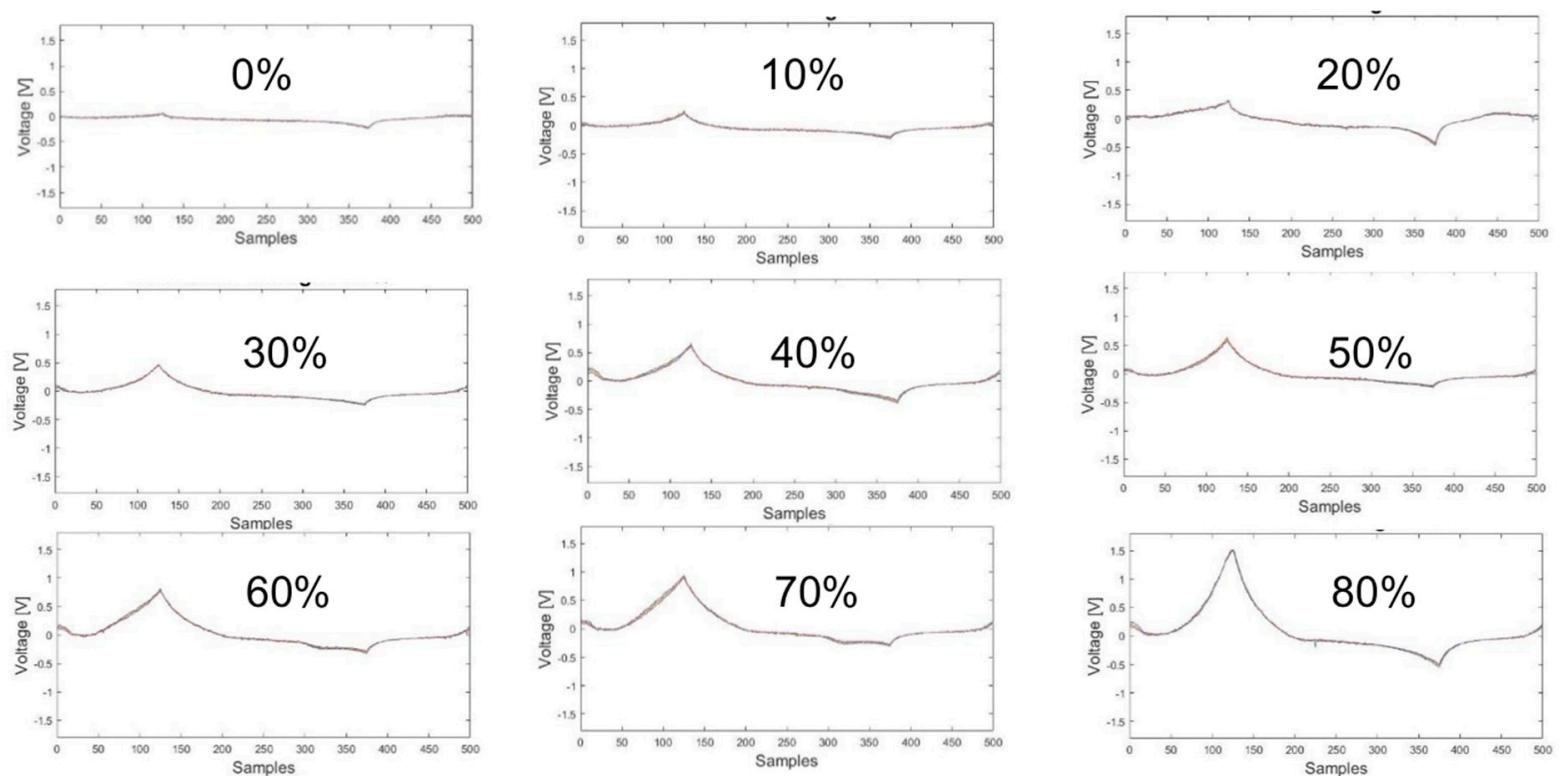

Figure 2. Output voltage signals registered by the system for increasing concentrations of $\mathrm{O}_{2}$, from $0 \%$ to $80 \%$. The range of interest for real application in the environment is round $20 \%$. The system is able to follow variation round $0.5 \%$ around this threshold.

\section{Conclusions and Future Developments}

The Comparison between the low-power BLE gas sensor here presented and its previous version (see Table 1) tells us that the good performance in terms of reproducibility, resolution and sensitivity are preserved.

In particular: size has been reduced of more than 4 times; supply voltage has been lowered of 3 times; power supply is $1 / 10$ of the starting one; time needed to take a measurement has been halved. Concerning the estimated limits of detection, it is worth noting that this is a pilot study, because the IAQ documents indicate threshold lower than the RMSECVs here reported [6]. Size and power reduction, together with BLE connectivity open the way to the utilization of this sensor in a wearable system including other devices and to the possibility of using them in a network with other systems. Moreover, sensor calibration must be challenged down to $10^{-1 \%}$ levels, in order to satisfy the IAQ requirements established in the official documents $[4,6]$. Looking at these indication by WHO (World Health Organization) and EU commission, it could be useful also to test these sensor for a series of other gas and pollutants. 
Table 1. Comparison between the previous [4] and the current version of the gas sensors in terms of size, supply voltage, power supply, wearability, measurement time and RMSECV with respect to $\mathrm{CO}_{2}$ and $\mathrm{O}_{2}$.

\begin{tabular}{lcc}
\hline & PREVIOUS VERSION [4] & CURRENT VERSION \\
\hline SIZE & $54 \mathrm{~cm}^{2}$ & $11.7 \mathrm{~cm}^{2}$ \\
SUPPLY VOLTAGE & $12 \mathrm{~V}$ & $3.6 \mathrm{~V}$ \\
POWER SUPPLY & $856 \mathrm{~mW}$ & $79.2 \mathrm{~mW}$ \\
WEARABILITY & $\mathrm{NO}$ & $\mathrm{SI}$ \\
MEASUREMENT & $150 \mathrm{~s}$ & $75 \mathrm{~s}$ \\
TIME & $1.97 \%$ & $3.39 \%$ \\
RMSECV CO & $2.16 \%$ & $0.47 \%$ \\
RMSECV $\mathrm{O}_{2}$ & & \\
\hline
\end{tabular}

Author Contributions: G.P. and M.S. conceived and designed the experiments; A.Z., F.M. and M.S. performed the experiments; L.V. realized device connectivity; S.G. and A.S. analyzed the data; A.D. and G.P. wrote the paper.

Acknowledgments: This work was partially funded by the 2016 FLAG-ERA project: (Frictionless Energy Efficient Convergent Wearables for Healthcare and Lifestyle Applications).

Conflicts of Interest: The authors declare no conflict of interest.

\section{References}

1. Bandodkar, A.J.; Wang, J. Non-invasive wearable electrochemical sensors: A review. Trends Biotechnol. 2014, 32, 363-371.

2. Gao, W.; Emaminejad, S.; Nyein, H.Y.; Challa, S.; Chen, K.; Peck, A.; Fahad, H.M.; Ota, H.; Shiraki, H.; Kiriya, D.; et al. Fully integrated wearable sensor arrays for multiplexed in situ perspiration analysis. Nature 2016, 529, 509.

3. Andreu-Perez, J.; Leff, D.R.; Ip, H.M.; Yang, G.Z.From wearable sensors to smart implants--toward pervasive and personalized healthcare. IEEE Trans. Biomed. Eng. 2015, 62, 2750-2762.

4. World Health Organization. WHO Guidelines for Indoor Air Quality: Selected Pollutants; World Health Organization: Geneva, Switzerland, 2010.

5. Pennazza, G.; Santonico, M.; Zompanti, A.; Parente, F.R.; Ferri, G.; D'Amico, A. Design and Development of an Electronic Interface for Gas Detection and Exhaled Breath Analysis in Liquids. IEEE Sens J. 2018, 18, 31-36, doi:10.1109/JSEN.2017.2771565.

6. Available online: http://publications.jrc.ec.europa.eu/repository/bitstream/JRC83683/eca\%20report\%2029_ final.pdf (accessed on October 2018).

(C) 2018 by the authors. Licensee MDPI, Basel, Switzerland. This article is an open access article distributed under the terms and conditions of the Creative Commons Attribution (CC BY) license (http://creativecommons.org/licenses/by/4.0/). 